\title{
The Effects of Vegetarian and Vegan Diets on Gut Microbiota
}

\author{
Aleksandra Tomova ${ }^{1 *}$, Igor Bukovsky ${ }^{2}$, Emilie Rembert ${ }^{3}$, Willy Yonas ${ }^{3}$, Jihad Alwarith ${ }^{3}$, \\ Neal D. Barnard ${ }^{3,4}$ and Hana Kahleova ${ }^{3}$ \\ ${ }^{1}$ Faculty of Medicine, Institute of Physiology, Comenius University in Bratislava, Bratislava, Slovakia, ${ }^{2}$ Center for Clinical \\ Nutrition, Bratislava, Slovakia, ${ }^{3}$ Physicians Committee for Responsible Medicine, Washington, DC, United States, ${ }^{4}$ Adjunct \\ Faculty, George Washington University School of Medicine and Health Sciences, Washington, DC, United States
}

The difference in gut microbiota composition between individuals following vegan or vegetarian diets and those following omnivorous diets is well documented. A plant-based diet appears to be beneficial for human health by promoting the development of more diverse and stable microbial systems. Additionally, vegans and vegetarians have significantly higher counts of certain Bacteroidetes-related operational taxonomic units compared to omnivores. Fibers (that is, non-digestible carbohydrates, found exclusively in plants) most consistently increase lactic acid bacteria, such as Ruminococcus, E. rectale, and Roseburia, and reduce Clostridium and Enterococcus species. Polyphenols, also abundant in plant foods, increase Bifidobacterium and Lactobacillus, which provide anti-pathogenic and anti-inflammatory effects and cardiovascular protection. High fiber intake also encourages the growth of species that ferment fiber into metabolites as short-chain fatty acids (SCFAs), including acetate, propionate, and butyrate. The positive health effects of SCFAs are myriad, including improved immunity against pathogens, blood-brain barrier integrity, provision of energy substrates, and regulation of critical functions of the intestine. In conclusion, the available literature suggests that a vegetarian/vegan diet is effective in promoting a diverse ecosystem of beneficial bacteria to support both human gut microbiome and overall health. This review will focus on effects of different diets and nutrient contents, particularly plant-based diets, on the gut microbiota composition and production of microbial metabolites affecting the host health.

Keywords: gut microbiota, fiber, nutrition, plant-based diet, postbiotics

\section{INTRODUCTION}

Recent studies of the human microbiome have emerged as an area of popular interest. For decades, many investigations have elucidated the impact of the human gut microbiota on the physiology of the host, with new and unexpectedly broad implications for health and disease.

The human microbiota, defined as the total of all microbial taxa associated with human beings (bacteria, viruses, fungi, protozoa, archaea), consists of a newly estimated $3 \times 10^{13}$ (trillion) microbes harbored by each person (1). The term microbiome is often incorrectly used interchangeably with the term microbiota. However, microbiome refers to the catalog of these microbes and their genes. The human gut microbiome represents $\sim 3.3$ million non-redundant microbial genes, which outnumbers the human genome of some 21,000 genes in the ratio of $\sim 150: 1$ (2). Interestingly, the diversity among the microbiomes of two different individuals is vast compared to their human genomic variation; humans are about $99.9 \%$ identical to each other in terms of their genome (3), but their gut microbiome can be up to $80-90 \%$ different (4). 
Recent advancements in laboratory techniques have revealed functions of the human gut microbiota related to immunity and the gastrointestinal, brain, and cardiovascular systems. Research has also suggested a profound effect of the human gut microbiota on host cells and genes. This extensive interaction has suggested that the microbiome functions effectively as a separate "organ."

Several studies have suggested that there are three basic bacterial enterotypes (5) (1) genus Prevotella (considered to be mostly anti-inflammatory and otherwise protective), (2) genus Bacteroides (more pro-inflammatory and possibly related to the heightened risk of metabolic syndrome and other pathological conditions), and (3) genus Ruminococcus (of which the biological significance is less evident) (6).

An imbalance of the gut microbiota has been linked with gastrointestinal conditions such as reflux, peptic ulcers, irritable bowel syndrome, non-alcoholic steatohepatitis, and inflammatory bowel disease. Additionally, some systemic conditions such as obesity, atherosclerosis, type 2 diabetes, cancer, Alzheimer's and Parkinson's disease, amyotrophic lateral sclerosis, autism spectrum disorder, atopy etc., also appear to be linked to unfavorable changes in gut microbiota composition (7-17). An accumulating body of evidence points to the gut microbiota as a mediator of dietary impact on the host metabolic status. Current research is focusing on the establishment of causal relationships in people and the development of therapeutic interventions such as personalized nutrition (18).

Dietary composition appears to have long-term and acute effects on the gut microbiota ecosystem $(19,20)$. Different longterm dietary patterns, such as vegetarian/vegan vs. omnivorous diets, have significant influence on gut microbiota composition. The different gut microbiota content is shown to provide different food nutrients metabolites, termed postbiotics. For instance, SCFAs, phytoestrogens, or isothiocyanates are more linked with the plant-based food, while TMAO and secondary bile acids with the meat-based diet. These and other postbiotics take part in the metabolism of the host in different ways. This review will focus on some general as well as specific aspects of this dynamic field of research.

\section{GUT MICROBIOTA: GENERAL ASPECTS}

In addition to bacteria, the gut is host to multiple kingdoms: archaea, viruses, and eukaryotes, including fungal species. The gut microbiota is represented by more than 1,000 microbial species, belonging primary to just two phyla: Bacteroidetes and Firmicutes (21). Based on human stool samples, overall, the genera Bacteroides, Prevotella, Bifidobacterium, Eubacterium, Clostridium, Streptococcus, and Enterobacteriaceae are most commonly found. It should be noted that stool samples provide reasonable estimations of the gut microbiota rather than a complete representation (22). This is because anaerobic species often attach to the gut mucosa, making it difficult to identify all bacterial species present in the large intestine. Also, it is probable that the biological significance of any genera or species is not given by its relative proportion in the whole ecosystem. Rather, its significance is observed through its metabolism/postbiotics and effects on the enteric nervous system, local immunity, brain, and genes.

\section{EFFECT OF DIET ON GUT MICROBIOTA COMPOSITION}

The difference in gut microbiota composition between individuals consuming a vegan/vegetarian and an omnivorous diet is well documented. Research shows that vegetarian/vegan diets foster different microbiota when compared to omnivores, with only a marginal difference between vegans and vegetarians (23). Changes in microbiota composition might be due to differences in bacteria directly consumed through food, differences in substrates consumed, variations in transit time through the gastrointestinal system, $\mathrm{pH}$, host secretion influenced by dietary patterns, and regulation of gene expression of the host himself and/or his/her microbiota (24).

A plant-based diet appears to be beneficial for human health by promoting the development of a more diverse gut microbial system, or even distribution of different species $(25,26)$.

\section{Diversity and Richness of the Gut Microbiota}

The diversity of the microbiota appears to have an important association with BMI, obesity, and arterial compliance; and a majority of the research suggests that a plant-based diet fosters a greater microbial diversity. Klimenko et al. found a positive association between alpha-diversity, or local microbial richness, and long-term fruit and vegetable intake $(p<0.05)$ (27). Likewise, Martinez et al. observed that adding whole-grain barley, brown rice, or a mixture of the first two to the diet of volunteers resulted in an increase in microbial diversity $(n=28)$ (28). Klimenko et al. also found a negative association between alpha-diversity and BMI $(p<0.05)(27)$.

However, a short-term dietary intervention advising increased fiber consumption resulted in a slight but significant decrease in diversity $(p<0.001)$. The researchers suggest this reduction in diversity might be the result of a rapid dietary change resulting in a temporary disruption to the microbial composition. This hypothesis of transitory microbial "stress" also explains the slight but significant increase in Enterobacteriaceae as a result of the intervention $(p<0.05)$. Enterobacteriaceae abundance is typically lower on a vegan diet vs. an omnivorous one $(P<$ $0.05)$ (29). This is likely due to the greater presence of butyrateproducing bacteria on a higher fiber diet, which can lower colonic $\mathrm{pH}$, preventing the growth of pathogenic bacteria, such as Enterobacteriaceae (30).

Verdam et al. observed reduced microbial diversity in obese vs. non-obese study participants $(n=28)$. The obese participants also displayed a reduction in the Bacteriodetes:Firmicutes ratio and an increase in Proteobacteria, a pro-inflammatory phylum. Likewise, an increase in C-reactive protein was observed $(p<0.001)$ which inversely correlated with the Bacteriodetes:Firmicutes ratio $(p<0.05)$. These observations suggest a pro-inflammatory effect of obesity-related microbiota (31). On the other hand, participants from the Adventist Health 
Study-2 $(60,903)$ following a vegan diet displayed the lowest BMI values when compared with those following a vegetarian or omnivorous diet (32). These findings indicate that a vegan diet, associated with lower body weight, might benefit microbial diversity and protect against inflammation.

Menni et al. observed that carotid-femoral pulse wave velocity, a measure of arterial stiffness, was negatively associated with microbiome diversity $(p=0.001)$ in women $(n=617)$ (33). This correlation remained significant after adjusting for insulin resistance and visceral fat. Arterial stiffness is oftentimes caused by hyperglycemia or hyperinsulinemia (34) and is significantly correlated with inflammatory adipokine levels. The researchers suggest the association between arterial stiffness and microbial diversity can be explained partially by the role of the gut in modulating systemic inflammation. Thus, an increase in microbial diversity might improve systemic inflammation, thereby reducing arterial stiffness.

Additionally, vegans and vegetarians have a significantly greater richness (alpha diversity) compared to omnivores, specifically counts of certain Bacteroidetes-related operational taxonomic units (OTUs) (35). It seems likely that many health benefits of vegetarian/vegan diets are, in part, mediated by the gut microbiota-not only through the higher relative abundance of those OTUs that are currently considered to be protective (Bacteroidetes, Prevotella, Roseburia, etc.), but also from postbiotic and epigenetic effects on various risk factors for chronic inflammation and chronic degenerative diseases (36).

\section{Effects of Diet on the Bacteroidetes:Firmicutes Ratio}

Despite significant inter-individual differences, a healthy adult intestinal microbiome is characterized by the dominance of these Bacteroidetes-related OTUs along with those of the Firmicutes phylum $(37,38)$. Research has shown variability in these phyla concentrations to be heavily affected by diet, specifically the ratio between the two when comparing omnivorous diets of the type common in North America, vs. a vegetarian/vegan diet. One study compared the bacterial composition between Indian $(n=11)$ and Chinese $(n=5)$ adults (39). While both populations ate diets centered around carbohydrates and vegetables, the Chinese diet was heavier in animal fat and protein than the Indian diet of whole grains and plant-based vegetarian foods. The percentage of Bacteroidetes within the microbiomes of Indian participants was nearly four times greater than in the Chinese, $16.39 \%$ vs. $4.27 \%$, respectively ( $p=0.001$ ). The higher abundance of Bacteroidetes in Indians was hypothesized to be due to their lower consumption of animal products; indicating a diet lower in animal products to be associated with greater Bacteroidetes counts.

Another study compared the fecal microbiota of Italian children $(n=15)$ vs. the fecal microbiota of children living in a rural western Africa, specifically in Burkina Faso $(n=14)(40)$. The Italian children typically consumed a Western diet, high in animal protein, sugar, starch, and fat and low in fiber. The African children of Burkina Faso consumed a diet low in fat and animal protein and rich in starch, fiber, and plant protein. The abundance of Firmicutes was twice as much in the Italian children than in the Burkina Faso children (63.7 vs. $27.3 \%$, respectively). The abundance of Bacteroidetes in the Italian children was less than half of that seen than in the Burkina Faso children (22.4 vs. 57.7\%, respectively). A decrease in Firmicutes levels, usually occurring in favor of Bacteroidetes and Bifidobacteria, as seen in response to an increase in resistant starches, may be beneficial in preventing and treating obesity (41). While these correlations between diet and microbiota composition are observed among different populations, it is important to consider other factors that may play a role, such as ethnicity, host genotypes, environmental factors, etc.

Research has shown that the balance of Bacteroidetes and Firmicutes is an important marker for obesity and higher BMI. Specifically, a decreased Bacteroidetes:Firmicutes ratio has a strong negative correlation with BMI $\left(r_{s}=0.59, P<\right.$ 0.001) (31). A possible explanation for this correlation may be found in the observation that a $20 \%$ increase in Firmicutes and a corresponding decrease in Bacteroidetes abundance is associated with a $150 \mathrm{kcal} /$ day increase in energy harvest, resulting in weight gain overtime. Therefore, an increased Bacteroidetes:Firmicutes ratio, as seen on a high fiber, plantbased diet, may result in weight loss by reducing the amount of energy extracted from the diet. Further research is needed to determine whether the increase in energy harvest due is due to the Bacteroidetes:Firmicutes ratio promoting adiposity or representing a host-mediated adaptive response to limit energy uptake (42).

Studies have also shown opposite trends in Firmicutes and Bacteroidetes. One study compared US children eating a Western diet to Bangladeshi children consuming a plant-based diet of rice, bread, and lentils. The Bacteroidetes:Firmicutes ratio was three times higher in the US children consuming the Western diet (43). This opposes the previous prediction of a Western diet resulting in a decreased Bacteroidetes:Firmicutes ratio. The researchers noted age and geographical differences as potential explanation for this departure from the expected ratio, as well as inter-subject variability. Another study asked participants to increase their fiber consumption and avoid Western diet foods. While prior studies would have suggested an increase in Bacteroidetes:Firmicutes, the ratio decreased $(0.13 \pm 0.2$ to $0.03 \pm 0.09$, Wilcoxon paired test $p<0.0001, n=430)$ (27). Another study analyzed the microbial composition of lean and obese subjects $(n=98)$ and observed that, when compared to lean subjects, overweight and obese volunteers presented a higher ratio Bacteroidetes to Firmicutes $(P=0.001$ and $P=0.005$, respectively) (44). Likewise, comparison of bacterial phyla did not show a significant difference in the Bacteroidetes to Firmicutes ratio between obese and lean volunteers $(n=20)$ (45). These examples reflect the difficulties in broadly linking certain phyla to particular diets. The primary challenge in analyzing specific microbiota is the need to consider the state and interaction dynamic of microbes encompassing the whole microbiome.

\section{Effects of Diet on Enterotypes}

As mentioned above, there are three main enterotypes observed in human microbiomes: Prevotella, Bacteroides, 
and Ruminococcus. Prevotella, a genus of the Bacteroidetes phyla, appears to be significantly richer in response to a vegan diet. In the previously mentioned study by De Filippo et al., Prevotella was exclusively present in the children of Burkina Faso consuming a diet low in fat and animal protein and rich in starch, fiber, and plant protein when compared to children living in Italy consuming a Western diet, high in animal protein, sugar, starch, and fat, and low in fiber (40). Another study compared the diets of 178 elderly residents living in either the community or in long-term residential care (46). The community group was found to consume a low to medium fat, high fiber diet; while the residents in long-term care consumed a moderate to high in fat, and low fiber diet. The study found that those in the community, eating a profile more reflective of a plant-based diet, more frequently had gut communities of the Prevotella enterotype.

The study comparing Indian and Chinese adults shows similar results (39). As expected, the Indians who were consuming less animal products and more plant-based foods than the Chinese had a significantly greater percentage of Prevotella (13.07 vs. $0.58 \%$, respectively). When the abundance of Prevotella was analyzed in Thai vegetarians vs. non-vegetarians, the vegetarians were found to have significantly higher numbers of Prevotella ( $p$ $=0.005$ ) (47). Other studies have shown vegan/vegetarian diets, high in plant-based foods, to be associated with high abundances of Prevotella $(48,49)$. This suggests additional support for greater Prevotella presence in those whom consume less animal products and more plant-based food. While mice studies suggest Prevotella to improve glucose metabolism by improving glycogen storage (50), the current lack of any additional research makes Prevotella merely a genus to describe an overall ecosystem of human gut bacteria, primarily under a plant-based diet.

Bacteroides, another main enterotype and genus of the Bacteroidetes phyla, also appears to be affected by diet but in a different way to Prevotella. Bacteroides has been positively correlated with long-term diets rich in animal protein and saturated fat $(20,27)$. This is likely due to their ability to tolerate bile, which is common in gut environments of those who consume animal products. In the study mentioned earlier comparing children in the US eating a Western diet vs. children in Bangladesh consuming a plant based diet, Bacteroides was the major genus in the US children's microbiota. High proportions of Bacteroides are found in the gut of humans consuming a Western diet and the opposite is found in those consuming a high fiber diet of fruits and legumes $(27,37,43,47,48)$.

Ruminococcus is the third major enterotype and is associated with long term fruit and vegetable consumption. Species of this genus are specialized in degrading complex carbohydrates, such as cellulose and resistant starch, found in plant based foods (51). These microbes degrade dietary fibers, producing butyrate, which acts as an anti-inflammatory compound. Ruminococcus is positively associated with low BMI and negatively associated with poor lipid profile (27). Likewise, abundance of Rumminococcus has been linked to lower endotoxemia and lower arterial stiffness, a predictor of cardiovascular risk (33). Walnut consumption has been significantly associated with enrichment of Ruminococcus as well (38). However, Ruminococcus has also been linked to low dietary fiber consumption in college students. While these microbes degrade complex carbohydrates, they also break down the resistant starches found in refined grain products (52). Ruminococcus might also play a role in the conversion of animal-derived choline to trimethylamine (TMA) (53). Thus, the abundance of Ruminococcus is influenced by both animal and plant based diets.

\section{Effects of Diet on Additional Bacteria}

While Bacteroides can be pro-inflammatory and their concentration is associated with long term consumption of animal products, a study analyzing 11 vegetarians, 20 vegans, and 29 omnivores (49) found a discrepancy in this generalization. In addition to finding Clostridium clostridioforme and Faecalibacterium prausnitzii, both considered to be health protective, in higher relative abundance in the vegetarians/vegans compared to the omnivores, Bacteroides thetaiotaomicron was also observed in higher abundance in these groups. This discrepancy in categorizing bacteria abundance under a plantbased diet vs. animal-based diet is not uncommon. Clostridium cluster XIVa was found in lower ratio in the vegetarian/vegans, contrary to a study showing Clostridium cluster XIVa bacteria to be a major component of gut microbiota in vegetarian women (103). Therefore, while generalizations can be made, some genus subtypes will be outliers. This discrepancy in some bacterial phyla in response to diet has been acknowledged by previous review papers and has been attributed to various reasons, such as different microbiome profiling methodologies, different host genetics, body mass index, and red wine and aspartame consumption $(54,55)$. These are all factors that have been shown to possibly modify our microbiota. Therefore, further studies are warranted in order to isolate their effects from those due to a plant based vs. omnivorous diet.

Taken together, dietary habits influence the composition of the intestinal microbiota. While dietary changes have a relatively fast impact (51) (within a week) on the microbial composition and consequently on its metabolites, these effects are modest and reversible (24). For example, changes of microbiota and immune parameters after a 3-month vegetarian diet are significant, but do not reflect the degree of change that occur with a long-term vegetarian diet (56).

\section{HOW PLANT FOOD COMPONENTS INFLUENCE GUT MICROBIOTA.}

\section{Nutrient Bioavailability}

Consuming food nutrients with low bioavailability has recently been found to be important. Lower nutrient bioavailability, found in larger food particles, intact plant cell walls, and food without thermal treatment, means that more nutrients reach lower in the gastrointestinal system, thus enriching nutrient delivery to the gut microbiota (57). This helps support normal gut microbiota development and function (57). Modern westernized diets contain more ultra-processed foods and acellular nutrients, or nutrients not containing cells. These components are more easily absorbed in the small intestine, depriving the colon of important nutrients, which may alter the composition and metabolism of the gut microbiota (58). Acellular food, e.g., sugar, has been 
shown to induce inflammation in young infants, adolescents, women of child-bearing age, and older adults. Whole plant foods have protective effects, favoring the growth of beneficial fiberdegrading bacteria in the colon (58).

\section{Carbohydrates}

Unlike digestible carbohydrates, non-digestible carbohydrates, such as resistance starch, and some sugars, reach the large intestine where they can be fermented by the gut microbiota to provide energy or produce postbiotics. However, both digestible and non-digestible carbohydrates may influence the gut microbiota. Digestible carbohydrates from fruits (e.g., glucose, sucrose, and fructose) have been shown to reduce Bacteroides and Clostridia (54). Non-digestible carbohydrates most consistently increase lactic acid bacteria, Ruminococcus, E. rectale, and Roseburia, and reduce Clostridium and Enterococcus species (54). Both digestible and non-digestible carbohydrates have been shown to increase Bifidobacteria, genus of the Actinobacteria phylum.

A study compared the Bifidobacteria levels in response to a randomized, double-blind, crossover trail. Participants consumed both a standard enteral formula and a formula supplemented with fructooligiosaccharides (FOS) and fiber (59) as a sole source of nutrition for 14 days. FOS and fiber are both forms of carbohydrates found naturally and abundantly in plant foods-bananas, artichokes, onion, etc. While the volume of formula prescribed was based on individual energy expenditures, a benchmark of 2,000 calories of the FOS/fiber formula provided $10.2 \mathrm{~g}$ of FOS and $17.8 \mathrm{~g}$ of fiber. The average daily intake of fermentable non-digestible carbohydrates is estimated to be $10 \mathrm{~g}$ from inulin and FOS (60). This amount does not include meals and products supplemented with inulin and FOS, which typically add an additional 3-10 g/portion. Therefore, $10.2 \mathrm{~g}$ of FOS in the formula is realistic for human consumption. $17.8 \mathrm{~g}$ of fiber in the formula is also realistic for human consumption as the average US male and female intake is $18 \mathrm{~g}$ and $15 \mathrm{~g}$, respectively ${ }^{1}$.

Bifidobacterium is a butyrate-producing genus known to play a protective role in the human gut barrier by providing defense against pathogens and diseases. When participants were given formulas with FOS and fiber, their Bifidobacteria increased from 5.1 to $26.6 \%(P=0.003)$ after 14 days. When formula was given without FOS and fiber, Bifidobacteria only increased from 3.3 to $8.6 \%(P=0.073)$. A negative correlation between baseline Bifidobacteria and magnitude of the bifidogenic effect was observed, indicating that those with lower initial amounts of Bifidobacteria benefit most from fructooligiosaccharides and fiber intake. In contrast, high consumption of cholesterol from animal products, was strongly associated with a lower abundance of Bifidobacteria (adj. $p=0.008$ ).

While these studies suggest that Bifidobacterium increase in response to a fiber-rich, high carbohydrate diet, other studies have shown conflicting results. One important confounding factor may be alcohol intake, which has been strongly associated with a lower abundance of Bifidobacteria (adj. $p=$

${ }^{1}$ https://www.ars.usda.gov/ARSUserFiles/80400530/pdf/dbrief/

12_fiber_intake_0910.pdf. (Accessed February 12, 2019)
0.006). Researchers comparing Bifidobacteria levels in vegans, vegetarians, and controls, found Bifidobacteria to be significantly lower $(p=0.002)$ in vegan samples than in controls eating a standard omnivorous diet. No difference between vegans and vegetarians was observed (29). Another study observed higher Bifidobacteria levels in meat eaters compared to participants who switched to a vegetarian diet for 4 weeks after eating a mixed Western diet, high in fat and meat (58). The relative decrease of Bifidobacterium in vegetarians and vegans may be explained by a relative abundance of other protective bacteria species, such as Prevotella. Prevotella has been observed confers antiinflammatory effects (40) and can decrease the growth of other bacteria by competing for fiber as an energy substrate (61).

A recent in vitro study elucidated the specific mechanism of action of carbohydrates, specifically selected dietary fibers, on gut microbiota. The study found the following fibers to have differing prebiotic effects: inulin, alpha-linked galacto-oligosaccharides, beta-linked galacto-oligosaccharides, xylo-oligosaccharides from corn cobs and high-fiber sugar cane, and beta-glucan from oats (62). Beta-glucan induced the growth of Prevotella and Roseburia with a concomitant increase in SCFA propionate production. Inulin and all oligosaccharides had a strong bifidogenic effect (62). This study also showed that all natural sugars, most notably non-digestible forms like inulin and oligosaccharides, increase SCFA levels (62). The prebiotic effects differ due to the type of bacteria that each fiber is broken down by. This is determined through bacterial specificity in which specific gene clusters within the bacterial genome dictate the saccharolytic enzymes that the bacteria can produce and, therefore, whether they can metabolize the prebiotic substrate (63). Non-digestible carbohydrates not only act as prebiotics by promoting the growth of beneficial microorganisms, but also reduce proinflammatory cytokine production, concentrations of serum triglycerides, total cholesterol, and LDL-cholesterol (54). Thus, non-digestible carbohydrates might confer protective effects against cardiovascular disease and central nervous system disorders.

\section{Proteins}

A majority of the studies have noted that protein consumption correlates positively with microbial diversity (54). However, animal and plant-proteins influence the gut microbiota in different ways. For instance, individuals consuming a high animal protein diet, from beef which is also high in fat, displayed lower abundances of bacteria, such as Roseburia, Eubacterium rectale, and Ruminococcus bromii, that metabolize dietary plant polysaccharides (51). Populations of bacteria that increase in response to a high animal protein diet when compared to subjects consuming a meatless diet are typically biletolerant microorganisms, such as Bacteroides and Clostridia (64). Additionally, a high-protein diet typically limits carbohydrate intake, which may lead to a decrease in butyrate-producing bacteria, and thereby to a proinflammatory state and an increased risk of colorectal cancer (65).

Individuals consuming pea protein exhibit increases in beneficial Bifidobacterium and Lactobacillus and decreases in pathogenic Bacteroides fragilis and Clostridium perfringens and, 
consequently increases in intestinal SCFA levels (54). Likewise, plant-derived proteins have been associated with lower mortality than animal-derived proteins (54).

\section{Fats}

Current evidence suggests that both the quantity and the quality of consumed fat significantly impact the gut microbiota composition (65).

A plant-based diet is generally naturally low in fat, which favors beneficial Bifidobacteria in human gut microbiota. The fat that does come from a vegan/vegetarian diet is made up of predominantly mono and polyunsaturated fats, which increase the Bacteroidetes:Firmicutes ratio, and on the genera level, increase lactic acid bacteria, Bifidobacteria and Akkermansia muciniphila (54). Nuts, particularly walnuts, have been shown to improve the gut microbiota by increasing Ruminococcaceae and Bifidobacteria, and decreasing Clostridium sp. cluster XIVa species (38).

On the other hand, saturated fat, found almost exclusively in animal sources, increases Bilophila and Faecalibacterium prausnitzii, and decreases Bifidobacterium (54). Some studies report that this change activates inflammation (induces proinflammatory cytokines such as IL-1, IL-6, and TNF- $\alpha$ ) and leads to metabolic disorders (66). High consumption of saturated and trans fat, predominately found in a Western diet, increases the risk of cardiovascular disease and reduces Bacteroidetes, Bacteroides, Prevotella, Lactobacillus ssp. and Bifidobacterium spp, and increases Firmicutes (40, 67).

N-3 polyunsaturated fatty acids, have been found to result in either no change to the microbiota, or beneficial increases in Bifidobacterium, Adlercreutzia, Lactobacillus, Streptococcus, Desulfovibrio, and Verrucomicrobia (Akkermansia muciniphila) $(54,67)$.

\section{Polyphenols}

Polyphenols, or naturally occurring plant metabolites (68), in plant foods increase Bifidobacterium and Lactobacillus abundance, which provide anti-pathogenic and antiinflammatory effects and cardiovascular protection (54). Common polyphenol-rich foods include fruits, seeds, vegetables, tea, cocoa products, and wine. For example, polyphenol extracts from tea generate an increase in Bifidobacterium and Lactobacillus-Enterococcus spp., which then yields an increased SCFA production on human microbiota in vitro (69).

\section{INFLUENCE OF MICROBIOME POSTBIOTICS ON HUMAN HEALTH}

Research on the gut-brain, gut-lung, and gut-liver axes highlights the importance of the microbiome on systemic human health. Studies note changes in central neural chemistry, inflammatory lung conditions, and non-alcoholic fatty liver disease pathogenesis with changes to microbial composition (70-72). The mechanism of communication among these organs stems from the microbial products and microbial metabolites of ingested nutrients. These products can be diet-independent (such as lipopolysaccharides, ribosomally synthesized post-translationally modified peptides etc.), but here we would like to describe a few examples of wellknown diet-dependent metabolites, such as SCFA and others. Depending on the bacteria and location along the intestinal tract, different bioactive molecules can be produced from different prebiotics and nutrients $(70,73)$. Microbial metabolites can have diverse positive health effects including local antiinflammatory and immunomodulatory effects, and systemic anti-obesogenic, antihypertensive, hypocholesterolemic, antiproliferative, and antioxidant effects (74). These postbiotic effects result from modulation of gene expression, metabolism, and intestinal functioning and depend on microbiota composition and substrates, largely dependent on diet.

\section{Short-Chain Fatty Acids}

SCFAs act as a substrate to maintain colonic epithelium, and are correlated with plant based food consumption (56). Maintenance of the intestinal barrier prevents endotoxemia and the subsequent inflammatory effects $(75,76)$. SCFAs acetate, propionate, and butyrate, are mostly microbial metabolites of fermented fiber and other carbohydrates, although a small fraction can derive from proteins. The fecal levels of these metabolites (and the corresponding esters) positively correlate with the consumption of fruits, vegetables, and legumes. Thus, their levels significantly increase in people who begin a plant-based diet. Interestingly, an increase in SCFAs is observed when omnivores consume a Mediterranean diet rich in fruit, legumes and vegetables (77).

While specific gut microbes are predisposed for SCFA production, different bacteria are known to produce different SCFAs. For example, enteric bacteria, such as Akkermansia muciniphila, Bifidobacterium spp., Prevotella spp., and Bacteroides spp. produce acetate; Bacteroides spp. produce propionate; and Coprococcus produces butyrate (78). The most butyrate producing bacteria are in Clostridium Cluster XIVa, IV, and XVI. These species are positively correlated with consumption of plant foods, and produce SCFAs that yield several health benefits.

The protective role of acetate, propionate and butyrate against different types of disease, such as type 2 diabetes, inflammatory bowel disease, and immune diseases, is well documented. For example, it has been shown that SCFAs promote immunity against pathogens (78), and are important components for microglia function and maturation and control of the bloodbrain barrier integrity (79). Other effect of SCFAs is to increase thermogenesis, preventing/treating obesity $(80,81)$. SCFAs serve as energy substrates for colonocytes, as well as for the body generally. For example, propionate serves as a gluconeogenic substrate in the liver and in the intestine (78).

Microbial interactions with dietary polysaccharides and the resulting SCFAs are important energy and signaling molecules. It is becoming increasingly accepted that butyrate-producing bacteria and butyrate, per se, may be beneficial for human health (78). Butyrate has been shown to play a key role in gut physiology as a major carbon source for colonocytes. It helps regulate critical functions of the intestine, such as intestinal motility, mucus production, visceral sensitivity, the epithelial barrier, immune homeostasis, and the mucosal oxygen gradient $(82,83)$. Thus, 
dietary fiber and carbohydrates can affect SCFA degradation while altering the abundance of the associated microbes. Taking together, diets rich in fiber might provide benefits to the intestine, as well as overall health.

\section{Phytoestrogens}

Phytoestrogens are plant-derived polyphenols that interact with estrogen receptors with either agonist or antagonist actions. A large majority of polyphenols are delivered to the gut, given their $1 \%$ bioavailability (57). The protective effects of plant polyphenols, particularly their anti-cancer, anti-inflammatory, and antioxidant effects, and their association with decreased risks of cardiovascular disease, obesity, diabetes, osteoporosis, and amyloid formation have been observed in humans (84-86). Increasing evidence shows that these effects are reached after bioactivation of the polyphenols by the gut microbiota $(87,88)$. Even though plant polyphenols have protective effects on human health, especially in the bioactive form, there is still a possibility of adverse effects due to their complexity of action and potential inter-individual variability (89).

While not all types of microbes participating in polyphenol metabolism are yet known, it has recently been shown that Bifidobacterium, Lactobacillus sp., Coriobacteriaceae, Clostridium sp., Bacteroides, and Saccharomyces yeast, are involved in the process of converting polyphenols to equol, urolithins, and enterolignans $(74,88)$. The qualitative and quantitative proportions of urolithins and equol produced correlate positively with the effects of phytoestrogens (88). Other bacterial species, such as Coriobacteriaceae and Eubacterium, are responsible for different polyphenol transformations (88).

The interaction of polyphenols and gut microbiota is bidirectional $(90,91)$. The gut bacteria produce microbial metabolites from polyphenols, which in turn serve as prebiotics for the gut bacteria. These metabolites, particularly urolithins, promote the growth of Lactobacillus and Bifidobacterium(88).

\section{Vitamins}

Gut microbiota are crucial for adequate vitamin levels in the human body. Menaquinone, folate, cobalamin, and riboflavin (ie: vitamins $\mathrm{K}, \mathrm{B} 9$, and B2) are produced by gut microbes (25). Different bacteria have biosynthetic properties for different vitamins, such as Bifidobacterium for vitamins $\mathrm{K}, \mathrm{B}_{12}$, biotin, folate, thiamine, Bacillus subtilis and Escherichia coli for riboflavin (92), and Lactobacillus for cobalamin and other B vitamins (93). The pathway analysis of the predicted metagenomes showed an enrichment of folate biosynthesis in vegans compared with omnivores (77).

\section{Isothiocyanates}

Isothiocyanates are compounds from glucosinolates, mainly found in plants, like cruciferous vegetables. Escherichia coli, certain Bacteroides, some Enterococcus, Lactobacillus agilis, certain Peptostreptococcus spp. and Bifidobacterium spp. metabolize glucosinolates to isothiocyanates, secreting their own myrosinase enzyme (94). These metabolites have cytoprotective and anti-oxidative effects through regulation of gene expression relating toneoplastic, atherosclerotic, and neurodegenerative processes (25).

\section{Aryl-Hydrocarbon Receptor Ligands}

Intestinal aryl-hydrocarbon receptor ligands are predominantly diet derived from plant food, specifically cruciferous vegetables. Through aryl-hydrocarbon receptors, the ligands act to promote intestinal immune function and gut homeostasis (95). Since arylhydrocarbon receptor ligands are gut microbiota-derived, any impairment to the gut microbiota, such as from a high-fat diet, can decrease aryl-hydrocarbon receptor ligands. In turn, this can cause gut inflammation and permeability and promote the development of metabolic syndrome, which can be improved by supplementation with a Lactobacillus strain (96). Additionally, a decrease in aryl-hydrocarbon receptors or ligands compromises the maintenance of intraepithelial lymphocytes and the control of the microbial load and composition, resulting in heightened immune activation and epithelial damage (95).

\section{Secondary Bile Acids and Coprostanol}

A separate group of postbiotics are cholesterol metabolites. Several bacterial strains, isolated from intestine or feces, are described to convert dietary or synthesized de novo cholesterol into coprostanol $(97,98)$, which is poorly absorbed by the human intestine. Thus, serum cholesterol in host is reduced, which decreases the risk of cardiovascular diseases. On the other hand, bile acids synthetized from cholesterol are converted by microbiota into secondary bile acids, found in different tissues and in feces. It is believed secondary bile acids are involved in the equilibrium of health/disease $(73,97)$. For example, they are associated with inflammatory bowel disease, liver and colon cancer.

\section{Trimethylamine N-Oxide (TMAO)}

Trimethylamine N-Oxide is a microbial metabolite believed to be associated with cardiovascular and neurological disorders. Carnitine and choline are the precursors of TMAO and are primarily found in foods of animal origin (eggs, beef, pork), with lower amounts found in beans and fish (99). Several microbial genera, like L-Ruminococcus, have been linked to the intake of animal proteins and fat and have been associated with TMAO levels (77). In general, meat intake appears to proliferate species of Bacteroides, Alistipes, Ruminococcus, Clostridia, and Bilophila, and decease Bifidobacterium. Higher TMAO levels have also been observed with red meat intake, increasing risk for cardiovascular disease and inflammatory bowel disease (54, 66). Vegetarians have a different gut microbiota composition than omnivores with a diminished capacity to produce trimethylamine (TMA), the precursor to TMAO. The plasma concentrations of TMAO appear to be similar in vegans and lacto-ovo-vegetarians $(99,100)$.

Lowering TMAO levels may be achieved through greater adherence to the Mediterranean diet, particularly a vegetarian one rich in fruits and vegetables $(77,100)$. Increased vegetable consumption reduces TMAO levels by reducing the enzymes responsible for converting TMA to TMAO and by remodeling the gut microbiota. The studies have shown TMAO production 
to decrease in vegetarians, which decreases their cardiovascular risk. To be objective, we have to mention a recent study, leaving a room for further analyses. Vegan fecal microbiota transplantation in metabolic syndrome patients resulted in significant changes in intestinal microbiota composition but failed to show changes in TMAO production. Authors explained that the 2-week follow-up was not a sufficient length of time to observe changes in TMAO production (101).

On average, twenty five percent of plasma metabolites are different between omnivores and vegans, suggesting a significant direct effect of diet on the host metabolome. No unique bacterial taxa have been significantly associated with individual metabolite levels after adjustment for multiple comparisons (102). These findings suggest that while inter-individual variability exists, dietary patterns significantly influence the microbial composition.

\section{CONCLUSION}

Current research indicates that diet is the essential factor for human gut microbiota composition, what in its turn is

\section{REFERENCES}

1. Sender R, Fuchs S, Milo R. Revised estimates for the number of human and bacteria cells in the body. PLoS Biol. (2016) 14:e1002533. doi: 10.1371/journal.pbio.1002533

2. Qin J, Li R, Raes J, Arumugam M, Burgdorf KS, Manichanh $\mathrm{C}$, et al. A human gut microbial gene catalogue established by metagenomic sequencing. Nature. (2010) 464:59-65. doi: 10.1038/nature 08821

3. Wheeler DA, Srinivasan M, Egholm M, Shen Y, Chen L, McGuire A, et al. The complete genome of an individual by massively parallel DNA sequencing. Nature. (2008) 452:872-6. doi: 10.1038/nature06884

4. Turnbaugh PJ, Hamady M, Yatsunenko T, Cantarel BL, Duncan A, Ley RE, et al. A core gut microbiome in obese and lean twins. Nature. (2009) 457:480-4. doi: 10.1038/nature07540

5. Arumugam M, Raes J, Pelletier E, Le Paslier D, Yamada T, Mende DR, et al. Enterotypes of the human gut microbiome. Nature. (2011) 473:174-80. doi: 10.1038/nature09944

6. Roager HM, Licht TR, Poulsen SK, Larsen TM, Bahl MI. Microbial enterotypes, inferred by the prevotella-to-bacteroides ratio, remained stable during a 6-month randomized controlled diet intervention with the new nordic diet. Appl Environ Microbiol. (2014) 80:1142-9. doi: 10.1128/AEM.03549-13

7. Bull MJ, Plummer NT. Part 1: The human gut microbiome in health and disease. Integr Med Clin J. (2014) 13:17-22.

8. Neish AS. Microbes in gastrointestinal health and disease. Gastroenterology. (2009) 136:65-80. doi: 10.1053/j.gastro.2008. 10.080

9. Ley RE, Turnbaugh PJ, Klein S, Gordon JI. Microbial ecology: human gut microbes associated with obesity. Nature. (2006) 444:1022-3. doi: $10.1038 / 4441022$ a

10. Larsen N, Vogensen FK, van den Berg FW, Nielsen DS, Andreasen AS, Pedersen BK, et al. Gut microbiota in human adults with type 2 diabetes differs from non-diabetic adults. PLoS ONE. (2010) 5:e9085. doi: 10.1371/journal.pone.0009085

11. Round JL, Mazmanian SK. The gut microbiota shapes intestinal immune responses during health and disease. Nat Rev Immunol. (2009) 9:313-23. doi: $10.1038 /$ nri2515 crucial for metabolizing nutrients into active for the host postbiotics. Up to date knowledge suggests that a plantbased diet may be an effective way to promote a diverse ecosystem of beneficial microbes that support overall health. Nonetheless, due to the complexity and interindividual differences, further research is required to fully characterize the interactions between diet, the microbiome, and health outcomes.

\section{AUTHOR CONTRIBUTIONS}

AT and IB contributed to conception and writing of the manuscript, ER, WY, JA, NB, and HK contributed and critically revised the manuscript. All authors approved the final manuscript.

\section{FUNDING}

This work was funded by PCRM and supported by the Grant Agency of Ministry of Education, Science, Research and Sport of the Slovak Republic VEGA 1/0286/18.

12. Spor A, Koren O, Ley R. Unravelling the effects of the environment and host genotype on the gut microbiome. Nat Rev Microbiol. (2011) 9:279-90. doi: 10.1038/nrmicro2540

13. Jiang C, Li G, Huang P, Liu Z, Zhao B. The gut microbiota and Alzheimer's Disease. J Alzheimers Dis JAD. (2017) 58:1-15. doi: 10.3233/JAD-161141

14. Unger MM, Spiegel J, Dillmann KU, Grundmann D, Philippeit H, Bürmann J, et al. Short chain fatty acids and gut microbiota differ between patients with Parkinson's disease and age-matched controls. Parkinsonism Relat Disord. (2016) 32:66-72. doi: 10.1016/j.parkreldis.2016.08.019

15. Ding HT, Taur Y, Walkup JT. Gut microbiota and autism: key concepts and findings. J Autism Dev Disord. (2017) 47:480-9. doi: 10.1007/s10803-016-2960-9

16. Jonsson AL, Bäckhed F. Role of gut microbiota in atherosclerosis. Nat Rev Cardiol. (2017) 14:79-87. doi: 10.1038/nrcardio.2016.183

17. Tomova A, Husarova V, Lakatosova S, Bakos J, Vlkova B, Babinska K, et al. Gastrointestinal microbiota in children with autism in Slovakia. Physiol Behav. (2015) 138:179-87. doi: 10.1016/j.physbeh.2014.10.033

18. Selber-Hnatiw S, Rukundo B, Ahmadi M, Akoubi H, Al-Bizri H, Aliu AF, et al. Human gut microbiota: toward an ecology of disease. Front Microbiol. (2017) 8:1265. doi: 10.3389/fmicb.2017.01265

19. Sonnenburg JL, Bäckhed F. Diet-microbiota interactions as moderators of human metabolism. Nature. (2016) 535:56-64. doi: 10.1038/nature18846

20. Wu GD, Chen J, Hoffmann C, Bittinger K, Chen YY, Keilbaugh SA, et al. Linking long-term dietary patterns with gut microbial enterotypes. Science. (2011) 334:105-8. doi: 10.1126/science.1208344

21. Lloyd-Price J, Abu-Ali G, Huttenhower C. The healthy human microbiome. Genome Med. (2016) 8:51. doi: 10.1186/s13073-016-0307-y

22. Yan W, Zheng J, Wen C, Ji C, Zhang D, Chen Y, et al. Efficacy of fecal sampling as a gut proxy in the study of chicken gut microbiota. bioRxiv. 2018:313577. doi: 10.1101/313577

23. Glick-Bauer M, Yeh MC. The health advantage of a vegan diet: exploring the gut microbiota connection. Nutrients. (2014) 6:4822-38. doi: 10.3390/nu6114822

24. Salonen A, de Vos WM. Impact of diet on human intestinal microbiota and health. Annu Rev Food Sci Technol. (2014) 5:239-62. doi: 10.1146/annurev-food-030212-182554

25. Derrien M, Veiga P. Rethinking diet to aid human-microbe symbiosis. Trends Microbiol. (2017) 25:100-12. doi: 10.1016/j.tim.2016.09.011 
26. Wong MW, Yi CH, Liu TT, Lei WY, Hung JS, Lin CL, et al. Impact of vegan diets on gut microbiota: an update on the clinical implications. Tzu Chi Med J. (2018) 30:200-3. doi: 10.4103/tcmj.tcmj_21_18

27. Klimenko NS, Tyakht AV, Popenko AS, Vasiliev AS, Altukhov IA, Ischenko DS, et al. Microbiome responses to an uncontrolled short-term diet intervention in the frame of the citizen science project. Nutrients. (2018) 10:E576. doi: 10.3390/nu10050576

28. Martínez I, Lattimer JM, Hubach KL, Case JA, Yang J, Weber CG, et al. Gut microbiome composition is linked to whole grain-induced immunological improvements. ISME J. (2013) 7:269-80. doi: 10.1038/ismej.2012.104

29. Zimmer J, Lange B, Frick JS, Sauer H, Zimmermann K, Schwiertz A, et al. A vegan or vegetarian diet substantially alters the human colonic faecal microbiota. Eur J Clin Nutr. (2012) 66:53-60. doi: 10.1038/ejcn.2011.141

30. Scott KP, Duncan SH, Flint HJ. Dietary fibre and the gut microbiota. Nutr Bull. (2008). 33: 201-211. doi: 10.1111/j.1467-3010.2008.00706.x

31. Verdam FJ, Fuentes S, de Jonge C, Zoetendal EG, Erbil R, Greve JW, et al. Human intestinal microbiota composition is associated with local and systemic inflammation in obesity. Obes Silver Spring Md. (2013) 21:E607615. doi: 10.1002/oby.20466

32. Orlich MJ, Fraser GE. Vegetarian diets in the Adventist Health Study 2: a review of initial published findings1234. Am J Clin Nutr. (2014) 100:353S-8S. doi: 10.3945/ajcn.113.071233

33. Menni C, Lin C, Cecelja M, Mangino M, Matey-Hernandez ML, Keehn L, et al. Gut microbial diversity is associated with lower arterial stiffness in women. Eur Heart J. (2018) 39:2390-7. doi: 10.1093/eurheartj/ehy226

34. Rizzoni D, Porteri E, Guelfi D, Muiesan ML, Valentini U, Cimino A, et al. Structural alterations in subcutaneous small arteries of normotensive and hypertensive patients with non-insulin-dependent diabetes mellitus. Circulation. (2001) 103:1238-44. doi: 10.1161/01.CIR.103.9.1238

35. Losasso C, Eckert EM, Mastrorilli E, Villiger J, Mancin M, Patuzzi I, et al. Assessing the Influence of Vegan, Vegetarian and Omnivore Oriented Westernized Dietary Styles on Human gut microbiota: a cross sectional study. Front Microbiol. (2018) 9:317. doi: 10.3389/fmicb.2018.00317

36. do Rosario VA, Fernandes R, Trindade EB. Vegetarian diets and gut microbiota: important shifts in markers of metabolism and cardiovascular disease. Nutr Rev. (2016) 74:444-54. doi: 10.1093/nutrit/nuw012

37. Simpson HL, Campbell BJ. Review article: dietary fibremicrobiota interactions. Aliment Pharmacol Ther. (2015) 42:158-79. doi: 10.1111/apt.13248

38. Bamberger C, Rossmeier A, Lechner K, Wu L, Waldmann E, Fischer $\mathrm{S}$, et al. A walnut-enriched diet affects gut microbiome in healthy caucasian subjects: a randomized, controlled trial. Nutrients. (2018) 10. doi: 10.3390/nu10020244

39. Jain $\mathrm{A}, \mathrm{Li} \mathrm{XH}, \mathrm{Chen} \mathrm{WN}$. Similarities and differences in gut microbiome composition correlate with dietary patterns of Indian and Chinese adults. AMB Express. (2018) 8:104. doi: 10.1186/s13568-018-0632-1

40. De Filippo C, Cavalieri D, Di Paola M, Ramazzotti M, Poullet JB, Massart $\mathrm{S}$, et al. Impact of diet in shaping gut microbiota revealed by a comparative study in children from Europe and rural Africa. Proc Natl Acad Sci USA. (2010) 107:14691-6. doi: 10.1073/pnas.1005963107

41. Martínez I, Kim J, Duffy PR, Schlegel VL, Walter J. Resistant starches types 2 and 4 have differential effects on the composition of the fecal microbiota in human subjects. PloS ONE. (2010) 5:e15046. doi: 10.1371/journal.pone.0015046

42. Ley RE, Bäckhed F, Turnbaugh P, Lozupone CA, Knight RD, Gordon JI. Obesity alters gut microbial ecology. Proc Natl Acad Sci USA. (2005) 102:11070-5. doi: 10.1073/pnas.0504978102

43. Lin A, Bik EM, Costello EK, Dethlefsen L, Haque R, Relman DA, et al. Distinct distal gut microbiome diversity and composition in healthy children from Bangladesh and the United States. PloS ONE. (2013) 8:e53838. doi: 10.1371/journal.pone.0053838

44. Schwiertz A, Taras D, Schäfer K, Beijer S, Bos NA, Donus C, et al. Microbiota and SCFA in lean and overweight healthy subjects. Obes Silver Spring Md. (2010) 18:190-5. doi: 10.1038/oby.2009.167

45. Andoh A, Nishida A, Takahashi K, Inatomi O, Imaeda H, Bamba S, et al. Comparison of the gut microbial community between obese and lean peoples using 16 S gene sequencing in a Japanese population. J Clin Biochem Nutr. (2016) 59:65-70. doi: 10.3164/jcbn.15-152

46. Claesson MJ, Jeffery IB, Conde S, Power SE, O'Connor EM, Cusack $\mathrm{S}$, et al. Gut microbiota composition correlates with diet and health in the elderly. Nature. (2012) 488:178-84. doi: 10.1038/nature 11319

47. Ruengsomwong S, La-Ongkham O, Jiang J, Wannissorn B, Nakayama J, Nitisinprasert S. Microbial community of healthy thai vegetarians and non-vegetarians, their core gut microbiota, and pathogen risk. J Microbiol Biotechnol. (2016) 26:1723-35. doi: 10.4014/jmb.1603. 03057

48. Jeffery IB, O’Toole PW. Diet-microbiota interactions and their implications for healthy living. Nutrients. (2013) 5:234-252. doi: 10.3390/nu5010234

49. Matijašić BB, Obermajer T, Lipoglavšek L, Grabnar I, Avguštin G, Rogelj I. Association of dietary type with fecal microbiota in vegetarians and omnivores in Slovenia. Eur J Nutr. (2014) 53:1051-64. doi: 10.1007/s00394-013-0607-6

50. Kovatcheva-Datchary P, Nilsson A, Akrami R, Lee YS, De Vadder F, Arora T, et al. Dietary fiber-induced improvement in glucose metabolism is associated with increased abundance of prevotella. Cell Metab. (2015) 22:971-82. doi: 10.1016/j.cmet.2015.10.001

51. David LA, Maurice CF, Carmody RN, Gootenberg DB, Button JE, Wolfe $\mathrm{BE}$, et al. Diet rapidly and reproducibly alters the human gut microbiome. Nature. (2014) 505:559-63. doi: 10.1038/nature 12820

52. Whisner CM, Maldonado J, Dente B, Krajmalnik-Brown R, Bruening M. Diet, physical activity and screen time but not body mass index are associated with the gut microbiome of a diverse cohort of college students living in university housing: a cross-sectional study. BMC Microbiol. (2018) 18:210. doi: 10.1186/s12866-018-1362-X

53. Ishii C, Nakanishi Y, Murakami S, Nozu R, Ueno M, Hioki K, et al. A metabologenomic approach reveals changes in the intestinal environment of mice fed on american diet. Int J Mol Sci. (2018) 19:E4079. doi: 10.3390/ijms19124079

54. Singh RK, Chang H-W, Yan D, Lee KM, Ucmak D, Wong K, et al. Influence of diet on the gut microbiome and implications for human health. J Transl Med. (2017) 15:73. doi: 10.1186/s12967-017-1175-y

55. Moschen AR, Wieser V, Tilg H. Dietary factors: major regulators of the Gut's microbiota. Gut Liver. (2012) 6:411-6. doi: 10.5009/gnl.2012. 6.4 .411

56. Zhang C, Björkman A, Cai K, Liu G, Wang C, Li Y, et al. Impact of a 3months vegetarian diet on the gut microbiota and immune repertoire. Front Immunol. (2018) 9:908. doi: 10.3389/fimmu.2018.00908

57. Ercolini D, Fogliano V. Food design to feed the human gut microbiota. J Agric Food Chem. (2018) 66:3754-8. doi: 10.1021/acs.jafc.8b00456

58. Zinöcker MK, Lindseth IA. The western diet-microbiome-host interaction and its role in metabolic disease. Nutrients. (2018) 10:3. doi: 10.3390/nu10030365

59. Whelan K, Judd PA, Preedy VR, Simmering R, Jann A, Taylor MA. Fructooligosaccharides and fiber partially prevent the alterations in fecal microbiota and short-chain fatty acid concentrations caused by standard enteral formula in healthy humans. J Nutr. (2005) 135:1896-902. doi: $10.1093 /$ jn/135.8.1896

60. Ten Bruggencate SJ, Bovee-Oudenhoven IM, Lettink-Wissink ML, Katan $\mathrm{MB}$, van der Meer R. Dietary fructooligosaccharides affect intestinal barrier function in healthy men. J Nutr. (2006) 136:70-4. doi: 10.1093/jn/136.1.70

61. Chen T, Long W, Zhang C, Liu S, Zhao L, Hamaker BR. Fiberutilizing capacity varies in Prevotella- versus Bacteroides-dominated gut microbiota. Sci Rep. (2017) 7:2594. doi: 10.1038/s41598-017-0 2995-4

62. Fehlbaum S, Prudence K, Kieboom J, Heerikhuisen M, van den Broek T, Schuren FHJ, et al. In vitro fermentation of selected prebiotics and their effects on the composition and activity of the adult gut microbiota. Int J Mol Sci. (2018) 19:10. doi: 10.3390/ijms19103097

63. Wilson B, Whelan K. Prebiotic inulin-type fructans and galactooligosaccharides: definition, specificity, function, and application in gastrointestinal disorders. J Gastroenterol Hepatol. (2017) 32:64-8. doi: 10.1111 /jgh. 13700

64. Hentges DJ, Maier BR, Burton GC, Flynn MA, Tsutakawa RK. Effect of a high-beef diet on the fecal bacterial flora of humans. Cancer Res. (1977) 37:568-71.

65. Sheflin AM, Melby CL, Carbonero F, Weir TL. Linking dietary patterns with gut microbial composition and function. Gut Microbes. (2016) 8:113-29. doi: 10.1080/19490976.2016.1270809 
66. Lee YK. Effects of diet on gut microbiota profile and the implications for health and disease. Biosci Microbiota Food Health. (2013) 32:1-12. doi: 10.12938/bmfh.32.1

67. Coelho OGL, Cândido FG, Alfenas RCG. Dietary fat and gut microbiota: mechanisms involved in obesity control. Crit Rev Food Sci Nutr. (2018) 2018:1-9. doi: 10.1080/10408398.2018.1481821

68. Pandey KB, Rizvi SI. Plant polyphenols as dietary antioxidants in human health and disease. Oxid Med Cell Longev. (2009) 2:270-8. doi: 10.4161/oxim.2.5.9498

69. Sun H, Chen Y, Cheng M, Zhang X, Zheng X, Zhang Z. The modulatory effect of polyphenols from green tea, oolong tea and black tea on human intestinal microbiota in vitro. J Food Sci Technol. (2018) 55:399-407. doi: 10.1007/s13197-017-2951-7

70. Klemashevich C, Wu C, Howsmon D, Alaniz RC, Lee K, Jayaraman A. Rational identification of diet-derived postbiotics for improving intestinal microbiota function. Curr Opin Biotechnol. (2014) 26:85-90. doi: 10.1016/j.copbio.2013.10.006

71. Cryan JF, O'Mahony SM. The microbiome-gut-brain axis: from bowel to behavior. Neurogastroenterol Motil. (2011) 23:187-92. doi: 10.1111/j.1365-2982.2010.01664.x

72. Compare D, Coccoli P, Rocco A, Nardone OM, De Maria S, Cartenì $M$, et al. Gut-liver axis: The impact of gut microbiota on nonalcoholic fatty liver disease. Nutr Metab Cardiovasc Dis. (2012) 22:471-6. doi: 10.1016/j.numecd.2012.02.007

73. Donia MS, Fischbach MA. Human microbiota. Small molecules from the human microbiota. Science. (2015) 349:1254766. doi: 10.1126/science. 1254766

74. Aguilar-Toalá JE, Garcia-Varela R, Garcia HS, et al. Postbiotics: An evolving term within the functional foods field. Trends Food Sci Technol. (2018) 75:105-14. doi: 10.1016/j.tifs.2018.03.009

75. Morrison DJ, Preston T. Formation of short chain fatty acids by the gut microbiota and their impact on human metabolism. Gut Microbes. (2016) 7:189-200. doi: 10.1080/19490976.2015.1134082

76. Cani PD, Bibiloni R, Knauf C, et al. Changes in gut microbiota control metabolic endotoxemia-induced inflammation in high-fat dietinduced obesity and diabetes in mice. Diabetes. (2008) 57:1470-81. doi: $10.2337 / \mathrm{db} 07-1403$

77. De Filippis F, Pellegrini N, Vannini L, Jeffery IB, La Storia A, Laghi L, et al. High-level adherence to a Mediterranean diet beneficially impacts the gut microbiota and associated metabolome. Gut. (2016) 65:1812-21. doi: 10.1136/gutjnl-2015-309957

78. Koh A, De Vadder F, Kovatcheva-Datchary P, Bäckhed F. From dietary fiber to host physiology: short-chain fatty acids as key bacterial metabolites. Cell. (2016) 165:1332-45. doi: 10.1016/j.cell.2016.05.041

79. Erny D, Hrabě de Angelis AL, Prinz M. Communicating systems in the body: how microbiota and microglia cooperate. Immunology. (2017) 150:7-15. doi: $10.1111 /$ imm. 12645

80. Reynés B, Palou M, Rodríguez AM, Palou A. Regulation of adaptive thermogenesis and browning by prebiotics and postbiotics. Front Physiol. (2019) 9:1908. doi: 10.3389/fphys.2018.01908

81. Canfora EE, Meex RCR, Venema K, Blaak EE. Gut microbial metabolites in obesity, NAFLD and T2DM. Nat Rev Endocrinol. (2019). doi: 10.1038/s41574-019-0156-z

82. Velázquez OC, Lederer HM, Rombeau JL. Butyrate and the colonocyte. Production, absorption, metabolism, and therapeutic implications. Adv Exp Med Biol. (1997) 427:123-34. doi: 10.1007/978-1-4615-5967-2_14

83. Borycka-Kiciak K, Banasiewicz T, Rydzewska G. Butyric acid - a well-known molecule revisited. Przeglad Gastroenterol. (2017) 12:83-9. doi: $10.5114 /$ pg.2017.68342

84. Panche AN, Diwan AD, Chandra SR. Flavonoids: an overview. J Nutr Sci. (2016) 5:e47, 1-15. doi: 10.1017/jns.2016.41

85. Ono K, Li L, Takamura Y, Yoshiike Y, Zhu L, Han F, et al. Phenolic compounds prevent amyloid $\beta$-protein oligomerization and synaptic dysfunction by site-specific binding. J Biol Chem. (2012) 287:14631-43. doi: 10.1074/jbc.M111.325456

86. Hossen MS, Ali MY, Jahurul MHA, Abdel-Daim MM, Gan SH, Khalil MI. Beneficial roles of honey polyphenols against some human degenerative diseases: a review. Pharmacol Rep. (2017) 69:1194-205. doi: 10.1016/j.pharep.2017.07.002
87. Landete JM, Arqués J, Medina M, Gaya P, de Las Rivas B, Muñoz R. Bioactivation of phytoestrogens: intestinal bacteria and health. Crit Rev Food Sci Nutr. (2016) 56:1826-43. doi: 10.1080/10408398.2013.789823

88. Tomás-Barberán FA, González-Sarrías A, García-Villalba R, Núñez-Sánchez MA, Selma MV, García-Conesa MT, et al. Urolithins, the rescue of "old" metabolites to understand a "new" concept: Metabotypes as a nexus among phenolic metabolism, microbiota dysbiosis, and host health status. Mol Nutr Food Res. (2017) 61:1. doi: 10.1002/mnfr.201500901

89. Rietjens IMCM, Louisse J, Beekmann K. The potential health effects of dietary phytoestrogens. Br J Pharmacol. (2017) 174:1263-80. doi: 10.1111/bph.13622

90. Cardona F, Andrés-Lacueva C, Tulipani S, Tinahones FJ, Queipo-Ortuño MI. Benefits of polyphenols on gut microbiota and implications in human health. J Nutr Biochem. (2013) 24:1415-22. doi: 10.1016/j.jnutbio.2013.05.001

91. Ozdal T, Sela DA, Xiao J, Boyacioglu D, Chen F, Capanoglu E. The reciprocal interactions between polyphenols and gut microbiota and effects on bioaccessibility. Nutrients. (2016) 8:2. doi: 10.3390/nu8020078

92. De Filippis F, Pellegrini N, Laghi L, Gobbetti M, Ercolini D. Unusual subgenus associations of faecal Prevotella and Bacteroides with specific dietary patterns. Microbiome. (2016) 4. doi: 10.1186/s40168-016-0202-1

93. LeBlanc JG, Milani C, de Giori GS, Sesma F, van Sinderen D, Ventura M. Bacteria as vitamin suppliers to their host: a gut microbiota perspective. Curr Opin Biotechnol. (2013) 24:160-8. doi: 10.1016/j.copbio.2012.08.005

94. Tian S, Liu X, Lei P, Zhang X, Shan Y. Microbiota: a mediator to transform glucosinolate precursors in cruciferous vegetables to the active isothiocyanates. J Sci Food Agric. (2018) 98:1255-60. doi: 10.1002/jsfa.8654

95. Li Y, Innocentin S, Withers DR, Roberts NA, Gallagher AR, Grigorieva $\mathrm{EF}$, et al. Exogenous stimuli maintain intraepithelial lymphocytes via aryl hydrocarbon receptor activation. Cell. (2011) 147:629-40. doi: 10.1016/j.cell.2011.09.025

96. Natividad JM, Lamas B, Pham HP, Michel ML, Rainteau D, Bridonneau $\mathrm{C}$, et al. Bilophila wadsworthia aggravates high fat diet induced metabolic dysfunctions in mice. Nat Commun. (2018) 9:2802. doi: 10.1038/s41467-018-05249-7

97. Gérard P. Metabolism of cholesterol and bile acids by the gut microbiota. Pathogens. (2013) 3:14-24. doi: 10.3390/pathogens3010014

98. Horáčková Š, Plocková M, Demnerová K. Importance of microbial defence systems to bile salts and mechanisms of serum cholesterol reduction. Biotechnol Adv. (2018) 36:682-90. doi: 10.1016/j.biotechadv.2017.12.005

99. Janeiro MH, Ramírez MJ, Milagro FI, Martínez JA, Solas M. Implication of trimethylamine N-Oxide (TMAO) in disease: potential biomarker or new therapeutic target. Nutrients. (2018) 10:10. doi: 10.3390/nu10101398

100. Obeid R, Awwad HM, Keller M, Geisel J. Trimethylamine-N-oxide and its biological variations in vegetarians. Eur J Nutr. (2017) 56:2599-609. doi: 10.1007/s00394-016-1295-9

101. Smits LP, Kootte RS, Levin E, Prodan A, Fuentes S, Zoetendal EG, et al. Effect of vegan fecal microbiota transplantation on carnitine- and cholinederived trimethylamine-N-oxide production and vascular inflammation in patients with metabolic syndrome. J Am Heart Assoc. (2018) 7:7. doi: 10.1161/JAHA.117.008342

102. Wu GD, Compher C, Chen EZ, Smith SA, Shah RD, Bittinger K, et al. Comparative metabolomics in vegans and omnivores reveal constraints on diet-dependent gut microbiota metabolite production. Gut. (2016) 65:63-72. doi: 10.1136/gutjnl-2014-308209

103. Hayashi H, Sakamoto M, Benno Y. Fecal microbial diversity in a strict vegetarian as determined by molecular analysis and cultivation. Microbiol Immunol. (2002) 46:819-31. doi: 10.1111/j.1348-0421.2002.tb02769.x

Conflict of Interest Statement: The authors declare that the research was conducted in the absence of any commercial or financial relationships that could be construed as a potential conflict of interest.

Copyright (c) 2019 Tomova, Bukovsky, Rembert, Yonas, Alwarith, Barnard and Kahleova. This is an open-access article distributed under the terms of the Creative Commons Attribution License (CC BY). The use, distribution or reproduction in other forums is permitted, provided the original author(s) and the copyright owner(s) are credited and that the original publication in this journal is cited, in accordance with accepted academic practice. No use, distribution or reproduction is permitted which does not comply with these terms. 